\title{
Reformulation of Some Finite Raytracing Equations Through Quadric Surfaces of Revolution
}

\author{
Ibrahim A. Sadiq \\ Department of Physics, College of Science, Al-Nahrain University, Jadryia, Baghdad-Iraq. \\ E-mail: abrahim742004@yahoo.com.
}

\begin{abstract}
This work exhibits analytical reformulation of four equations of finite raytracing through quadric surfaces of revolution. This reformulation enhances comprehending the procedure of finite raytracing firmly besides it shows how to obtain these formulas. The equations reformulated in this work clarified that the corresponding ones in [1] were merely typographically incomplete equations.
\end{abstract}

Keywords: Quadric Surfaces of Revolution, Raytracing, Skew Raytracing.

\section{Introduction}

Finite raytracing is essentially a sequence of successive refraction and transfer calculations based on solid geometry and Snell's law [1]. It is a method for tracing rays in three dimensions by using solid geometry to get an exact analysis for ray tracing [2]. This method takes into account the most general type of rays known as the skew rays; that's why it is also called skew raytracing. The meaning of skew rays is those which are not co-planar with axis (optical axis) [3].

In my attempt to construct a programming code to trace skew rays [1] had been adopted as the considered reference to make use of. This adoption based on the compatibility between my research needs and the features of [1]. The program code for tracing skew rays through spherical surfaces was functional whilst the one for tracing skew rays through quadric surfaces of revolution was not. This led to reconsidering the raytracing equations thoroughly and to start figuring out the equations malfunctioned the program for tracing skew rays through quadric surfaces.

Although [1] is a highly selective, selfcontained, and well-sketched text book with very solid notation and as clear as elegant approach, errata have been found. Some of those were equations for finite raytracing.

Not Like many other text books, [1] not only referred to the final mathematical formulas but also mentioned how to obtain those formulas and omitted their derivations to meet the needs of its purpose as it had been intended.
Thanks to mathematically firm approach for deriving the raytracing equations, [1] used, besides the vector analysis notation, [1] employed, I was able to reformulate those typographically incomplete equations for finite raytracing with no fear of misleading. These typographically incomplete equations for tracing skew rays through quadric surfaces in [1] were:

1) $\Delta=\frac{F}{G+\sqrt{G^{2}-c F\left(I+(\varepsilon-1) N^{2}\right)}}$

2) $\alpha, \beta, \gamma=\frac{1-c x,-c y, 1-c \varepsilon z}{\sqrt{1-2 c(\varepsilon-1) z}}$

3) $\cos I=\frac{N-c(L x+M y+N \varepsilon z)}{\sqrt{1-2 c(\varepsilon-1) z}}$

4) $\cos I=\frac{\sqrt{G^{2}-c F\left(1+(\varepsilon-1) N^{2}\right.}}{\sqrt{1-2 c(\varepsilon-1) \Delta N}}$

[1] pointed out that equation (4.28) can be obtained by using the same manner approached to derive $\Delta$ for raytracing through spherical surfaces. Thus, it was necessary to follow the derivation of $\Delta$ through spherical surfaces before reformulating equation (4.28) where $F$ and $G$ are defined by:

$F=c\left(x_{o}^{2}+y_{o}^{2}\right) \ldots \ldots . .$.
$G=N-c\left(L x_{o}+M y_{o}\right)$

and

$L^{2}+M^{2}+N^{2}=1$ 
Derivation of $\Delta$ through Spherical Surfaces

The approach of derivation is quoted from [1] starts with using (4.5) into (4.6)

$$
\left.\begin{array}{l}
x=x_{o}+L \Delta \\
y=y_{o}+M \Delta \\
z=N \Delta
\end{array}\right\} \ldots \ldots . . .
$$

yields:

$$
\begin{aligned}
N \Delta= & \frac{c}{2}\left[x_{o}^{2}+2 x_{o} L \Delta+L^{2} \Delta^{2}+y_{o}^{2}+\right. \\
& \left.2 y_{o} M \Delta+M^{2} \Delta^{2}+N^{2} \Delta^{2}\right] \\
N \Delta= & \frac{1}{2} c\left(x_{o}^{2}+y_{o}^{2}\right)+\frac{2}{2} c\left(L x_{o}+M y_{o}\right) \Delta+ \\
& \frac{c}{2}\left(L^{2}+M^{2}+N^{2}\right) \Delta^{2}
\end{aligned}
$$

The latter equation in terms of equations (4.9) and (4.18) can be written as:

$$
\begin{aligned}
& N \Delta=\frac{F}{2}+c\left(L x_{o}+M y_{o}\right) \Delta+\frac{c}{2} \Delta^{2} \\
& 0=\frac{F}{2}-\left[N-c\left(L x_{o}+M y_{o}\right)\right] \Delta+\frac{c}{2} \Delta^{2}
\end{aligned}
$$

Now, the latter equation in terms of the equation (4.10) becomes:

$$
\frac{c}{2} \Delta^{2}-G \Delta+\frac{F}{2}=0
$$

which is similar to $A x^{2}+B x+$ Const. $=0$ that is solved by $x=\frac{-B \pm \sqrt{\left(B^{2}-4 \times A \times \text { Const. }\right)}}{2 \times A} \quad$ It is

$$
\text { clear }
$$

that

$$
A=\frac{c}{2} \quad, \quad B=-G \quad, \quad \text { Const. }=\frac{F}{2}
$$

and $c \Delta=G \pm \sqrt{G^{2}-c F}$

Now by multiplying the R.H.S of the latter equation with the negative sign by the quantity $\left(G+\sqrt{G^{2}-c F}\right)$ and dividing result by the same quantity yields [1]:

$$
\Delta=\frac{F}{G+\sqrt{G^{2}-c F}}
$$

\section{Equation (4.28) Reformulation}

By following the same manner to solve for $\Delta$ through spherical surfaces illustrated in the previous section. Applying (4.5) into (4.25) $z=\frac{c}{2}\left(x^{2}+y^{2}+\varepsilon z^{2}\right)$

$$
\left.\begin{array}{l}
x=x_{o}+L \Delta \\
y=y_{o}+M \Delta \\
z=N \Delta
\end{array}\right\}
$$

yields

$$
\begin{aligned}
& N \Delta=\frac{c}{2}\left[\left(x_{o}+L \Delta\right)^{2}+\left(y_{o}+M \Delta\right)^{2}+\varepsilon N^{2} \Delta^{2}\right] \\
& N \Delta=\frac{c}{2}\left[\left(x_{o}^{2}+y_{o}^{2}\right)+2\left(L x_{o}+M y_{o}\right) \Delta+\right. \\
& \left.\quad L^{2} \Delta^{2}+M^{2} \Delta^{2}+\varepsilon N^{2} \Delta^{2}\right] \\
& N \Delta=\frac{c}{2}\left(x_{o}^{2}+y_{o}^{2}\right)+c\left(L x_{o}+M y_{o}\right) \Delta+ \\
& \quad \frac{c}{2}\left(L^{2}+M^{2}+\varepsilon N^{2}\right) \Delta^{2} \\
& \frac{c}{2}\left(L^{2}+M^{2}+\varepsilon N^{2}\right) \Delta^{2}-\left[N-c\left(L x_{o}+M y_{o}\right)\right] \Delta+ \\
& \frac{c}{2}\left(x_{o}^{2}+y_{o}^{2}\right)=0
\end{aligned}
$$

Now, the latter equation in terms of equations (4.9), and (4.10) becomes:

$$
\frac{c}{2}\left(L^{2}+M^{2}+\varepsilon N^{2}\right) \Delta^{2}-G \Delta+\frac{F}{2}=0
$$

Using (4.18) $L^{2}+M^{2}=1-N^{2}$

Using the latter expression, the equation of $\Delta$ becomes:

$$
\begin{aligned}
& \frac{c}{2}\left(1-N^{2}+\varepsilon N^{2}\right) \Delta^{2}-G \Delta+\frac{F}{2}=0 \\
& \frac{c}{2}\left[1+(\varepsilon-1) N^{2}\right] \Delta^{2}-G \Delta+\frac{F}{2}=0
\end{aligned}
$$

solving for $\Delta$ gives:

$$
\begin{gathered}
A=\frac{c}{2}\left[1+(\varepsilon-1) N^{2}\right], B=-G, \quad \text { Const. }=\frac{F}{2} \\
c \Delta=\frac{G \pm \sqrt{G^{2}-4 * \frac{c}{2}\left[1+(\varepsilon-1) N^{2}\right] * \frac{F}{2}}}{\left[1+(\varepsilon-1) N^{2}\right]} \\
c \Delta=\frac{G \pm \sqrt{G^{2}-c F\left(1+(\varepsilon-1) N^{2}\right)}}{\left[1+(\varepsilon-1) N^{2}\right]}
\end{gathered}
$$

Now by multiplying the R.H.S of the latter equation with the negative sign by the quantity $\left(G+\sqrt{G^{2}-c F\left(1+(\varepsilon-1) N^{2}\right.}\right)$ and dividing the result by the same quantity yields:

$$
\Delta=\frac{F}{G+\sqrt{G^{2}-c F\left(1+(\varepsilon-1) N^{2}\right)}}
$$

\section{Equation (4.30) Reformulation}

The approach for deriving the components of the unit normal at the point of incidence, [1] illustrated, starts with rewriting the equation of the surface 
Journal of Al-Nahrain University

Science

$z=\frac{c}{2}\left(x^{2}+y^{2}+\varepsilon z^{2}\right)$

into the form

$F(x, y, z)=0$

And finally obtained the direction cosines of the normal (components of the unit normal) by using

$$
\alpha, \beta, \gamma=\frac{-\left[\left(\frac{\partial F}{\partial x}, \frac{\partial F}{\partial y}, \frac{\partial F}{\partial z}\right)\right]}{\sqrt{\left(\frac{\partial F}{\partial x}\right)^{2}+\left(\frac{\partial F}{\partial y}\right)^{2}+\left(\frac{\partial F}{\partial z}\right)^{2}}}
$$

Now, we shall proceed as mentioned above. Equation (4.25) in the form of (4.20) can be expressed as:

$F=x^{2}+y^{2}+\varepsilon z^{2}-2 r z=0$

where $c=1 / r$, so the latter expression can be expressed as:

$c x^{2}+c y^{2}+c \varepsilon z^{2}-2 z=0$

$c\left(x^{2}+y^{2}\right)=2 z-c \varepsilon z^{2}$ Differentiate the latter as (4.20) requires

$$
\left.\begin{array}{l}
\left.\begin{array}{l}
\frac{\partial F}{\partial x}=2 c x \\
\frac{\partial F}{\partial y}=2 c y \\
\frac{\partial F}{\partial z}=2 c \varepsilon z-2
\end{array}\right\} \\
\left(\frac{\partial F}{\partial x}\right)^{2}=4 c^{2} x^{2} \\
\left(\frac{\partial F}{\partial y}\right)^{2}=4 c^{2} y^{2} \\
\left(\frac{\partial F}{\partial x}\right)^{2}+\left(\frac{\partial F}{\partial y}\right)^{2}=4 c^{2}\left(x^{2}+y^{2}\right) \\
\left(\frac{\partial F}{\partial z}\right)^{2}=4 c^{2} \varepsilon^{2} z^{2}-8 c \varepsilon z+4
\end{array}\right\}
$$

Now, the denominator of (4.24) will be expressed in terms of the $z$ parameter only

$$
\begin{aligned}
& \sqrt{\left(\frac{\partial F}{\partial x}\right)^{2}+\left(\frac{\partial F}{\partial y}\right)^{2}+\left(\frac{\partial F}{\partial z}\right)^{2}} \\
& =\sqrt{4 c\left(2 z-c \varepsilon z^{2}\right)+4 c^{2} \varepsilon^{2} z^{2}-8 c \varepsilon z+4} \\
& =2 \sqrt{2 c z-c^{2} \varepsilon z^{2}+c^{2} \varepsilon^{2} z^{2}-2 c \varepsilon z+1} \\
& =2 \sqrt{1+2 c(1-\varepsilon) z+c^{2}(\varepsilon-1) \varepsilon z^{2}} \\
& =2 \sqrt{1-2 c(\varepsilon-1) z+c^{2} \varepsilon(\varepsilon-1) z^{2}}
\end{aligned}
$$

In terms of (BB) and (CC), equation (4.24) becomes

$$
\alpha, \beta, \gamma=\frac{-[2 c x, 2 c y, 2 c \varepsilon z-2]}{2 \sqrt{1-2 c(\varepsilon-1) z+c^{2} \varepsilon(\varepsilon-1) z^{2}}}
$$

$$
\alpha, \beta, \gamma=\frac{-[c x, c y, c \varepsilon z-1]}{\sqrt{1-2 c(\varepsilon-1) z+c^{2} \varepsilon(\varepsilon-1) z^{2}}}
$$

or

$$
\left.\begin{array}{l}
\alpha=\frac{-c x}{\sqrt{1-2 c(\varepsilon-1) z+c^{2} \varepsilon(\varepsilon-1) z^{2}}} \\
\beta=\frac{-c y}{\sqrt{1-2 c(\varepsilon-1) z+c^{2} \varepsilon(\varepsilon-1) z^{2}}} \\
\gamma=\frac{1-c \varepsilon z}{\sqrt{1-2 c(\varepsilon-1) z+c^{2} \varepsilon(\varepsilon-1) z^{2}}}
\end{array}\right\}
$$

where (DD) is the reformulated components of the normal vector

\section{Equation (4.31) Reformulation}

[1] stated that "the cosine of the angle of incidence $(\cos I)$ at the point of incidence can be obtained by the scalar multiplication of (4.30) with the direction cosines $(L, M, N)$ of the ray as in the following equation:

$\cos I=\alpha \cdot L+\beta \cdot M+\gamma \cdot N$

Now, by applying the reformulated equation labeled by (DD) above into (4.42) yields:

$$
\cos I=\frac{-c x L-c y M+N-c \varepsilon z N}{\sqrt{1-2 c(\varepsilon-1) z+c^{2} \varepsilon(\varepsilon-1) z^{2}}}
$$

With little rearrangement, the reformulated equation can be expressed as:

$$
\cos I=\frac{N-c(L x+M y+N \varepsilon z)}{\sqrt{1-2 c(\varepsilon-1) z+c^{2} \varepsilon(\varepsilon-1) z^{2}}}
$$

\section{Equation (4.32) Reformulation}

This equation is also incomplete. This was so; because it has been derived form (4.31) but in terms of (4.25). We shall proceed reformulating it by applying (4.5) into the formulated (4.31) which is $(\mathrm{EEH})$.

$$
\begin{aligned}
& \left.\begin{array}{l}
x=x_{o}+L \Delta \\
y=y_{o}+M \Delta \\
z=N \Delta
\end{array}\right\} \ldots \ldots \ldots \ldots \ldots \ldots \ldots \ldots \ldots \ldots \ldots \ldots \ldots \ldots \ldots \ldots \ldots \ldots \ldots \ldots \ldots \ldots \ldots \ldots \\
& \cos I=\frac{N-c\left[L\left(x_{o}+L \Delta\right)+M\left(y_{o}+M \Delta\right)+N \varepsilon(N \Delta)\right]}{\sqrt{1-2 c(\varepsilon-1) N \Delta+c^{2} \varepsilon(\varepsilon-1)(N \Delta)^{2}}} \\
& \cos I=\frac{\left[N-c\left(L x_{o}+M y_{o}\right)\right]-c \Delta\left[L^{2}+M^{2}+\varepsilon N^{2}\right)}{\sqrt{1-2 c(\varepsilon-1) N \Delta+c^{2} \varepsilon(\varepsilon-1)(N \Delta)^{2}}}
\end{aligned}
$$

In terms of (4.10), and (4.18) and

$$
L^{2}+M^{2}=1-N^{2}
$$

the equation of $\cos I$ becomes: 
$\cos I=\frac{G-c \Delta\left(1+(\varepsilon-1) N^{2}\right)}{\sqrt{1-2 c(\varepsilon-1) N \Delta+c^{2} \varepsilon(\varepsilon-1)(N \Delta)^{2}}}$

The latter equation in terms of (AA) becomes:

$$
\cos I=\frac{\sqrt{G-c F\left(1+(\varepsilon-1) N^{2}\right)}}{\sqrt{1-2 c(\varepsilon-1) N \Delta+c^{2} \varepsilon(\varepsilon-1)(N \Delta)^{2}}}
$$

This Equation represents the reformulation of (4.32).

\section{Results and Discussion}

The results are the reformulated equations:

1. $\Delta=\frac{F}{G+\sqrt{G^{2}-c F\left(1+(\varepsilon-1) N^{2}\right)}} \quad$ (AA) which represents the reformulation of (4.28). In the notation of [1], the parameter $I$ stands for the angle the incident ray makes with normal of the surface. Thus, the parameter $I$ inside the square root of (4.28) should be replaced by 1 .

2. The equations (DD) and (EE)

$$
\begin{aligned}
& \left.\begin{array}{l}
\alpha=\frac{-c x}{\sqrt{1-2 c(\varepsilon-1) z+c^{2} \varepsilon(\varepsilon-1) z^{2}}} \\
\beta=\frac{-c y}{\sqrt{1-2 c(\varepsilon-1) z+c^{2} \varepsilon(\varepsilon-1) z^{2}}} \\
\gamma=\frac{1-c \varepsilon z}{\sqrt{1-2 c(\varepsilon-1) z+c^{2} \varepsilon(\varepsilon-1) z^{2}}}
\end{array}\right\} \\
& \cos I=\frac{N-c(L x+M y+N \varepsilon z)}{\sqrt{1-2 c(\varepsilon-1) z+c^{2} \varepsilon(\varepsilon-1) z^{2}}}
\end{aligned}
$$

represent the reformulation of equations (4.30) and (4.31) respectively in which the third term including $c^{2} z^{2}$ in the denominator was missing.

3. The equation

$$
\cos I=\frac{\sqrt{G-c F\left(1+(\varepsilon-1) N^{2}\right)}}{\sqrt{1-2 c(\varepsilon-1) N \Delta+c^{2} \varepsilon(\varepsilon-1)(N \Delta)^{2}}}
$$

represents the reformulation of (4.32) in which the term $c^{2} \varepsilon(\varepsilon-1)(N \Delta)^{2}$ was missing.

\section{Conclusion}

The analytical reformulation achieved in this work is based on the firm approach [1] illustrated; and this emphasizes that equations (4.28), (4.30), (4.31), and (4.32) were typographically incomplete equations.

\section{References}

[1] W. T. Welford; "Aberrations of the Symmetrical Optical Systems"; Academic Press, NY;(1974).

[2] C.S. William and O. A. Becklund; "Optics: A Short Course for Engineers and Scientists"; John- Wiley and Sons (1972).

[3] Max Born and Emile Wolf; "The Principles of Optics, $5^{\text {th }}$ edition"; Pergamon Press (1975)

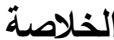

هذا العمل يعرض إعادة صياغة تحليلية لأربعة من معادلات الأقتفاء الاقيق للأشعة البصرية خلال سطوح معادلات الدرجة الثانية. إن إعادة الصياغة هذه تعزز فهم طريقة العمل في إقتفاء الأشعة البصرية بالأضافة الى إنها

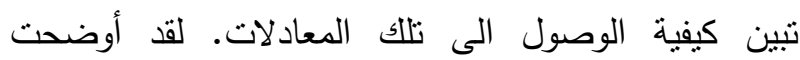
المعادلات التي صيغت في هذا العمل أن المعادلات المقابلة لها في [1] كانت مجرد معادلات غير مكتملة من الناحية

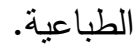

